\title{
Universiteit
}

Leiden

The Netherlands

\section{Autobiographical memory after acute stress in healthy young men}

Tollenaar, M.S.; Elzinga, B.M.; Spinhoven, P.; Everaerd, W.

\section{Citation}

Tollenaar, M. S., Elzinga, B. M., Spinhoven, P., \& Everaerd, W. (2009). Autobiographical memory after acute stress in healthy young men. Memory, 17, 301-310. Retrieved from https://hdl.handle.net/1887/15009
Version:
Not Applicable (or Unknown)
License:
Leiden University Non-exclusive license
Downloaded from: $\quad$ https://hdl.handle.net/1887/15009

Note: To cite this publication please use the final published version (if applicable). 


\title{
Autobiographical memory after acute stress in healthy young men
}

\author{
Marieke S. Tollenaar, Bernet M. Elzinga, and Philip Spinhoven \\ Leiden University, The Netherlands \\ Walter Everaerd \\ University of Amsterdam, The Netherlands
}

\begin{abstract}
Autobiographical memories have been found to be less specific after hydrocortisone administration in healthy men, resembling memory deficits in, for example, depression. This is the first study to investigate the effects of stress-induced elevated cortisol levels on autobiographic memory specificity and experience in healthy young men. Autobiographical memories were elicited by neutral and negative cue words, with instructions to recall either recent or remote memories. No effect of psychosocial stress was found on memory specificity or experience, but cortisol increases tended to be related to less specific, recent memories elicited by neutral cue words, especially when participants were physically aroused during memory retrieval. These results indicate that autobiographical memories are fairly resistant to an acute stressor in healthy young men, but that endogenous cortisol increases might be related to autobiographical memory retrieval. More research into the relation between endogenous cortisol increases and autobiographic memory retrieval is needed, especially in stress-related disorders.
\end{abstract}

Keywords: Autobiographical memory; Stress; Cortisol; Memory specificity; Memory retrieval; Depression.

Dependent on the memory stage being tested, acute stress and high cortisol levels can have impairing or enhancing effects on memory. While encoding and consolidation are found to be facilitated by cortisol (e.g., Buchanan \& Lovallo, 2001), retrieval and working memory are found to be impaired by acute stress (Elzinga \& Roelofs, 2005; Kuhlmann, Piel, \& Wolf, 2005b; Oei, Everaerd, Elzinga, Van Well, \& Bermond, 2006; Tollenaar, Elzinga, Spinhoven, \& Everaerd, 2008) or exogenous cortisol administration (Het, Ramlow, \& Wolf, 2005; Lupien, Gillin, \& Hauger, 1999; Wolf et al., 2001). Recent studies suggest that the impairing effects of cortisol on human memory may be mediated by reduced prefrontal and medial temporal lobe (MTL) activation (de Quervain et al., 2003; Oei et al., 2007). Animal studies have shown that the impairing effects of cortisol on memory are mediated by hippocampal and prefrontal glucocorticoid receptors (Lupien \& LePage, 2001), and are dependent on noradrenergic signalling of the basolateral nucleus of the amygdala (Roozendaal, Hahn, Nathan, de Quervain, \& McGaugh, 2004; Roozendaal, McReynolds, \& McGaugh, 2004). In line with the hypothesis that noradrenergic activation is a

Address correspondence to: M. S. Tollenaar, Leiden University, Institute for Psychological Research, Clinical, Health and Neuropsychology Unit, PO Box 9555, 2300 RB Leiden, The Netherlands. E-mail: mtollenaar@fsw.leidenuniv.nl

We would like to thank Hans Sandberg, Reineke Bos, Jitske Tiemensma, Josje Mulder, Inge van der Neut, and Dagmar Feenstra for their help in conducting the laboratory stressor and processing the data. We thank Hans van Pelt and Jolanda Verhagen for the cortisol analyses at the Chemical Clinical lab of the LUMC. We also like to thank the anonymous reviewers for their helpful comments. The research of M. S. Tollenaar is supported by grant 400-03-210 from the Dutch Organisation for Scientific Research (NWO). 
prerequisite for cortisol effects on memory, recent studies in humans have shown that this effect is dependent on arousal elicited by the encoded stimuli and/or the environment (de Quervain, Aerni, \& Roozendaal, 2007; Elzinga \& Roelofs, 2005; Tollenaar et al., 2008).

Declarative memory retrieval in humans is most often tested with word tasks, using free recall, cued recall, or recognition paradigms (see also Wolf, 2008), showing fairly consistent results. However, laboratory word tasks are not necessarily an ecologically valid measure of real-life memories of one's personal past, defined as autobiographical memory (Tulving, 2002). Furthermore, disorders such as acute stress disorder, depression, or post traumatic stress disorder (PTSD) that are characterised by cortisol disregulations have been related to recall of nonspecific, over-general autobiographic memories (e.g., Bryant, Sutherland, \& Guthrie, 2007; Harvey, Bryant, \& Dang, 1998; Kangas, Henry, \& Bryant, 2005; Williams \& Scott, 1988).

Based on the hierarchical model of autobiographical memory by Conway and PleydellPearce (2000), one might expect that if retrieval of memory details is impaired by stress and/or cortisol, autobiographic memory retrieval might not progress to the level of event-specific knowledge and remain over-general after stress exposure or cortisol administration. Based on this same model, Williams et al. (2007) suggested three mechanisms that may underlie over-general autobiographical memories, described in the CaRFAX model. Important in the present context is that each of these processes can potentially be affected by stress or cortisol increases: First of all, specific memory retrieval requires cognitive resources and hence impaired executive functioning might lead to over-general memory retrieval (Dalgleish et al., 2007). As stress and cortisol have been found to impair working memory (i.e., an indicator of executive functioning capacity), this might thus lead to the recall of less-specific memories. Second, decreased specificity might be a result of functional avoidance. Because stress may induce negative mood states (e.g., Kuhlmann et al., 2005b), stressed individuals might try to avoid further mood disturbances by avoidance of sensory and perceptual details of negative events and hence adopt an over-general retrieval style (see Au Yeung, Dalgleish, Golden, \& Schartau, 2006, and Svaldi \& Mackinger, 2003, for the negative effects of mood inductions on memory specificity). Third, rumination about self-referential attributes can also lead to lowered specificity. While ruminating, the search process might not progress to specific memories but rather move across the memory hierarchy by retrieving abstract, self-related conceptual knowledge (see also Spinhoven, Bockting, Kremers, Schene, \& Williams, 2007). During a psychosocial stress test (such as the Trier Social Stress Test, one that is frequently used to induce psychosocial stress), participants are confronted with their performance in a social situation. This might lead to increased activation of self-schemas and rumination, leading to less-specific memory retrieval. In sum, based on the hierarchical model by Conway and Pleydell-Pearce (2000) and the CaRFAX model by Williams et al. (2007), we might expect that increases in stress and cortisol lead to overgeneral memory retrieval.

The only experimental study so far in which the effects of cortisol administration on the retrieval of autobiographical memories have been examined is that by Buss, Wolf, Witt, and Hellhammer (2004). They found that acute cortisol administration in healthy young men diminished recall of specific memories, especially in response to neutral cue words. Interestingly, the fact that it was mostly neutral autobiographical memories that were impaired by cortisol administration is not in line with previous studies using word recall in which the retrieval of emotional words was found to be most affected by cortisol (Kuhlmann, Kirschbaum, \& Wolf, 2005a) and stress (Kuhlmann et al., 2005b; Tollenaar et al., 2008; Wolf, 2008).

The effects of acute stress and endogenously increased cortisol levels on autobiographical memory have never been studied before. Based on the predictions from the CaRFAX model, stress exposure might affect even more processes involved with the retrieval of specific memories than cortisol administration. The present study therefore investigated the effects of an acute psychosocial stressor and related cortisol increases on autobiographical memory retrieval in healthy young men. Autobiographical memory specificity and subjective emotional experience of the memories were tested, elicited by means of both neutral and negative cue words. In addition, to test whether both recent and remote memories are equally vulnerable to stress, participants were instructed to recall half of the memories from 
childhood, and the other half from the 2 years before the test.

We thus expect the psychosocial stress task and its related cortisol increases to reduce autobiographical memory specificity. As a consequence, we also expect that subjective emotional experience of the memories will be rated as less intense. From the literature on the effects of cortisol on declarative memory retrieval we would expect mostly negative, emotional memories to be affected, although the study by Buss et al. (2004) found impairing effects of cortisol on neutral autobiographical memories. However, on the basis of predictions derived from the CaRFAX model, we expect effects of stress on memory specificity for both neutral and negative cue words.

\section{METHOD AND MATERIALS}

\section{Participants}

A total of 40 healthy male participants between the age of 18 and $30(M=21.7, S D=3.4)$ were recruited at Leiden University for the present study. Females were not included in the study because of confounding effects of gender (e.g., by menstrual cycle and contraceptive pills) on cortisol responses (Kirschbaum, Wust, \& Hellhammer, 1992). Inclusion criteria were no reported medical or psychological problems in the past year, no reported use of medication, and no drug or alcohol abuse. There were no differences between the stress and control group on depressive and anxious symptoms as measured with the Beck Depression Inventory-II (BDI-II; van der Does, 2002) stress group: $M=8.45, S D=1.39$; control group: $M=6.45, S D=0.97, F(1,38)=$ $1.40, p=.25$, and the Hospital Anxiety and Depression Scales (HADS; Spinhoven et al., 1997) HADS depression: stress group: $M=2.65$, $S D=0.53$; control group: $M=2.35, S D=0.49$, $F(1,38)=0.17, p=.68$; HADS anxiety: stress group: $M=4.90, S D=0.49$; control group: $M=$ $5.10, S D=0.63, F(1,38)=0.06, p=.80$. Participants gave written informed consent before participation and were rewarded with either money or course credits afterwards. The study was approved by the Ethics Committee of the Faculty of Social and Behavioral Sciences at Leiden University.

\section{Procedure}

To avoid confounding of cortisol measurements, participants were instructed not to drink any caffeinated drinks on the morning of the study, and further not to smoke or eat, and to only drink water, an hour before the start of the study. All testing sessions took place in the afternoon, starting either at $12 \mathrm{pm}$ or $3 \mathrm{pm}$. Participants were randomly assigned to either a stress $(N=$ $20)$ or control $(N=20)$ condition in a betweenparticipants design. At the start of the test session the autobiographical memory task (AMT) was practised and the first physiological measurement was taken $(t=0$ minutes $)$. During the stress and control task the experimenter was seated behind a one-way screen. After the stress task and a short break, the second physiological measurement was taken $(\mathrm{t}=30$ minutes). The AMT was administered by the experimenter directly after this measurement and lasted on average 30 minutes ( $M=31.8$ minutes, $S D=6.14$ minutes). This was followed by the last physiological measurement $(t=60$ minutes $)$.

\section{Measures}

Autobiographical memory task. Autobiographical memory was measured with an adapted version of the AMT (Williams \& Broadbent, 1986). Participants were given six negative and six neutral cue words (see Appendix) and were asked to produce a specific memory to each cue word. Specific memories were described as events that lasted less than a day and occurred at a particular time and place. As a restriction, for each valence category participants were instructed to name three remote memories (from their primary school time) and three recent memories (from the last 2 years, except for the current day). Four pseudo-random versions with valence and date instructions were constructed. Cue words were presented on a card and read aloud by the experimenter. Participants were prompted at least once to elaborate on their memory. If there was no response after 60 seconds "no memory" was reported. Memories were tape-recorded for later scoring. After each memory a questionnaire on emotional experience and the age of the participant during the memory was administered (see below). Reliability of the memories was not 
verified. For scoring, only the first answer was used. Memories were only scored as specific when the reported event did not last longer than a day (i.e., an extended memory) and was not a repeated event (i.e., a categoric memory). Semantic associations (e.g., "I often feel sad") and memories from the wrong time period (which happened only four times in total) were also classified as not specific. Memory specificity was scored by a trained rater blind to condition. A random sample of $20 \%$ of all memories was double-scored by an independent rater, leading to an inter-rater agreement of more than $93 \%$ (Cohen's kappa $=0.82$ ). Maximum specificity score for the total AMT was 12, with 3 points for each category (negative-remote/negativerecent/neutral-remote/neutral-recent).

To examine subjective emotional experience of the memories and to verify the valence of the memories a Dutch questionnaire was used, derived from the study by Greenberg et al. (2005) on emotional valence, intensity (arousal and physical feelings) and feelings of re-experiencing (reliving, seeing in mind, coherence, remembering-knowing).

Stress protocol. Psychosocial stress was induced using the Trier Social Stress Task (TSST), which is well known for inducing hypothalamic-pituitaryadrenal (HPA) axis and cardiovascular responses in a large part of participants (see Kirschbaum, Pirke, \& Hellhammer, 1993, for a detailed procedure). The TSST consists of three parts each of about 5 minutes: an anticipation/preparation period, followed by a public speech task, and a cognitive task (in the present study an arithmetic task and a working memory task) in front of an audience of three people with a camera and voice recorder. The control group had to write a letter for a fictitious job interview and also performed the working memory task, but with no audience present.

Physiological measures. Cortisol saliva samples were obtained at 0,30 , and approximately 60 minutes with reference to the stress task with Salivette collection devices (Sarstedt, Germany). Saliva samples were stored at $-20^{\circ} \mathrm{C}$ before assay. Free cortisol saliva levels were determined with a competitive electrochemiluminescence immunoassay ECLIA, using a Modular Analytics E170 immunoassay analyser (Roche Diagnostics, Mannheim, Germany). The functional detection limit was $2.0 \mathrm{nmol} / \mathrm{l}$ and the intra- and inter-assay variability coefficients in the measuring range were less than $10 \%$. The analytical detection limit was $0.5 \mathrm{nmol} / 1$ and values below $0.5 \mathrm{nmol} / \mathrm{l}$ were not reported. Heart rate, blood pressure, and subjective stress measures were taken at 0,15 (during), 30, and 60 minutes with reference to the stress task using the Omron R5-I. Subjective stress experience was measured with visual analogue scales, ranging from $0 \mathrm{~mm}$ to $100 \mathrm{~mm}$, on tension, mood, and tiredness.

\section{Statistical analyses}

Repeated measures ANOVAs were performed to test the effect of the stress task on the physiological measures and on memory specificity and experience. Condition (stress vs control) was set as a between-participants variable and valence of the cue words (negative vs neutral) and time period (remote vs recent) as within-participants factors. Memory experience was tested with two multivariate dependent variables: emotional intensity (arousal and physical feelings) and feelings of re-experiencing (reliving, seeing in mind, coherence, remembering/knowing). To study the effects of cortisol increases on memory specificity, areas under the curve increase (AUCi) for cortisol with respect to baseline $(t=0)$ were calculated (using the equation from Pruessner, Kirschbaum, Meinlschmid, \& Hellhammer, 2003) and Spearman Rank correlations were performed. The overall $\alpha$ was set at $5 \%$.

\section{RESULTS}

\section{Stress outcomes}

All physiological measures showed an increase in the stress group over time compared to the control group (see Figure 1a-d) cortisol: $F(2,76)=13.59, p<.001$, heart rate: $F(3,102)=$ $7,97, p<.001$, systolic blood pressure: $F(3,99)=$ 19.42, $p<.001$, diastolic blood pressure: $F(3$, $99)=13.5, p<.001$. Cortisol levels and the stress-induced increases of cortisol did not differ between groups that started at $12 \mathrm{pm}$ or $3 \mathrm{pm}-$ control group: $F(2,36)=0.23, p=.78$; stress group: $F(2,36)=0.62, p=.54$. The stress group did not report more subjective tension, sadness, or tiredness after the stress task than the control group (all $p \mathrm{~s}>.35$ ). 

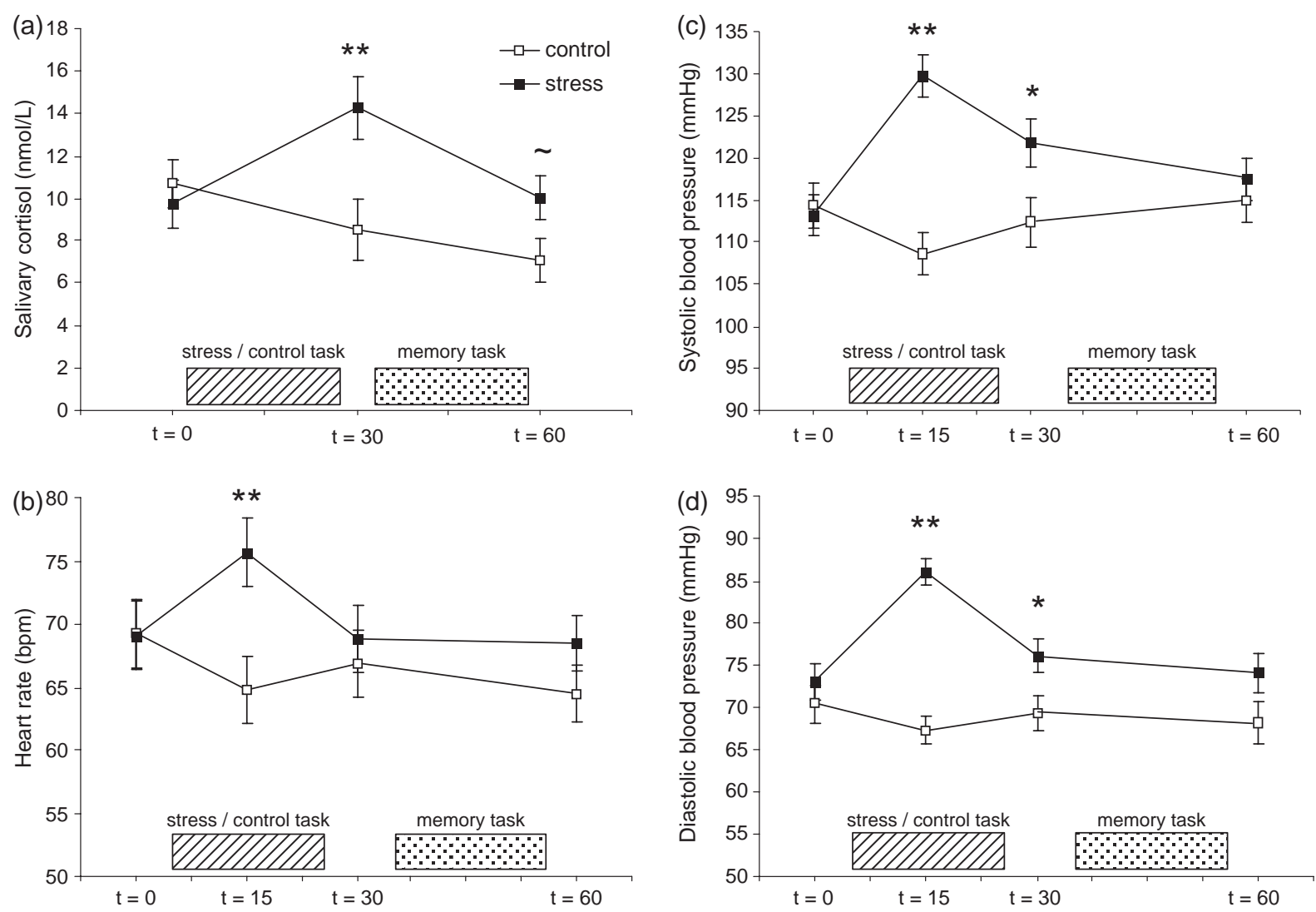

Figure 1. Mean $( \pm \mathrm{SEM})$ levels of (a) free salivary cortisol (nmol/L), (b) heart rate (bpm), (c) systolic, and (d) diastolic blood pressure $(\mathrm{mmHg})$ levels from the start till the end of the test session. $* *=p<.01, *=p<.05, \sim=p<.10$.

\section{Memory outcomes}

No differences were found between the stress and the control group in the number of specific memories retrieved, $F(1,38)=0.25, p=.62$ (see

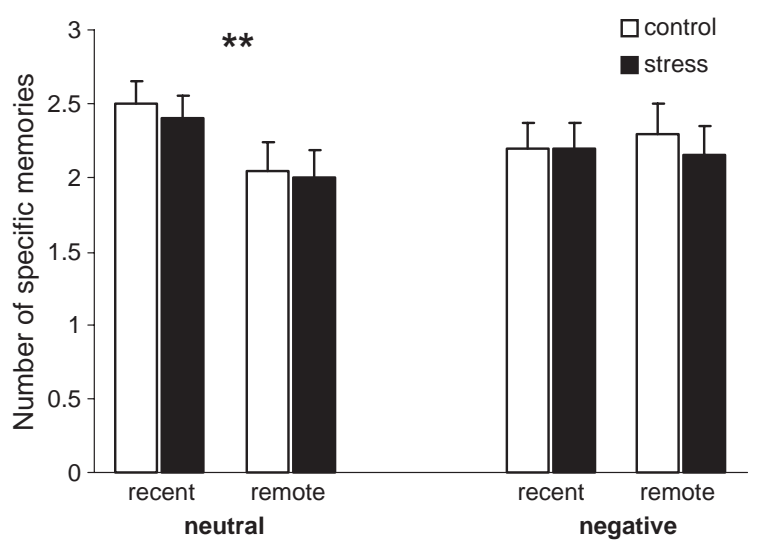

Figure 2. Mean number $( \pm \mathrm{SEM})$ of specific neutral and negative autobiographical memories retrieved by the stress and control group, divided over time period (recent or remote). $* *=$ Significant difference between recent and remote neutral memories $(p<.01)$.
Figure 2). No interactions between condition and valence or time period were found either, $F(1$, $38)=0.00, p=1.00 ; F(1,38)=0.52, p=.82$. Including time of day (12 pm or $3 \mathrm{pm})$ as a covariate did not change these outcomes (all $p \mathrm{~s}>.56$ ). The repeated measures ANOVA did reveal a time period by valence interaction, $F(1,38)=4.31, p<$ .05. Post hoc analyses showed that within the neutral valence category, remote memories were less specific than recent memories, $F(1,38)=8.14$, $p<.01$, while the specificity of remote and recent negative memories did not differ, $F(1,38)=0.025$, $p=.88$. Overall, there were no differences in the specificity of neutral and negative memories, $F(1,38)=0.034, p=.86$.

To control for depressive and anxious symptoms, the scores on the BDI-II and HADS were entered as covariates in the above analyses. These covariates did not affect the main and interaction effects of condition-condition: $F(1,35)=0.99$, $p=.33$; condition by valence: $F(1,35)=0.03$, $p=.88$; condition by time period: $F(1,35)=$ $0.01, p=.92$. 
TABLE 1

Spearman rank correlations

\begin{tabular}{|c|c|c|c|}
\hline Cue: & & $\begin{array}{c}\text { Stress } \\
(\mathrm{N}=20)\end{array}$ & $\begin{array}{c}\text { Stress }(\mathrm{N}=14) \text { with } \\
\text { heightened SBP at } t=60\end{array}$ \\
\hline \multirow[t]{2}{*}{ Neutral } & Remote & $-0.06(.82)$ & $-0.20(.49)$ \\
\hline & Recent & $-0.39(.09)^{\dagger}$ & $-0.67(.008)^{* *}$ \\
\hline \multirow[t]{2}{*}{ Negative } & Remote & $-0.27(.25)$ & $-0.22(.46)$ \\
\hline & Recent & $-0.04(.85)$ & $-0.15(.60)$ \\
\hline
\end{tabular}

Spearman rank correlations, $R h o(\rho)$, between increases in cortisol due to stress and memory specificity on the AMT. $\mathrm{SBP}=$ systolic blood pressure, ${ }^{* *}=p<.01,{ }^{\dagger}=p<.10$.

Memory experience was also not affected by stress. Both the repeated measures MANOVA for emotional intensity and re-experiencing did not show any main effects for condition, $F(2$, $35)=0.53, p=.59 ; \quad F(4,33)=1.34, p=.28$, or any interaction effects with time period or valence (all $p \mathrm{~s}>.10$ ). Overall, negative memories were rated as more emotional intense, $F(2$, $35)=33.25, p<.001$, more negatively valenced, $F(1,37)=300.79, \quad p<.001$, and with higher re-experiencing scores than neutral memories, $F(4, \quad 33)=6.09, \quad p=.001$. Recent memories tended to be rated as more emotional, $F(2$, $35)=3.13, p=.06$, and were rated with higher re-experiencing scores than remote memories, $F(4,33)=11.34,<.001$.

\section{Cortisol and memory specificity}

No significant correlations were found between memory specificity and cortisol increase (AUCi) within the stress group (see Table 1, left column), although there was a trend for a negative correlation between AUCi cortisol increase and specificity of recent, neutral memories (rho $=-0.39$, $p=.09$, see also Figure 3a). Since previous studies have shown that cortisol effects on memory are dependent on arousal elicited by the memory or by the environment (de Quervain et al., 2007; Tollenaar et al., 2008), we calculated these correlations in the group of participants that showed heightened sympathetic arousal until the end of the memory task (at $t=60)$, indicated by a systolic blood pressure higher than at baseline $(N=14$, see Table 1 , right column). When participants from the stress group were physically aroused until the end of the AMT, the correlation between cortisol increase and specificity of recent, neutral memories was indeed stronger $(r h o=-0.67, p<.01$, see also Figure $3 b)$. When
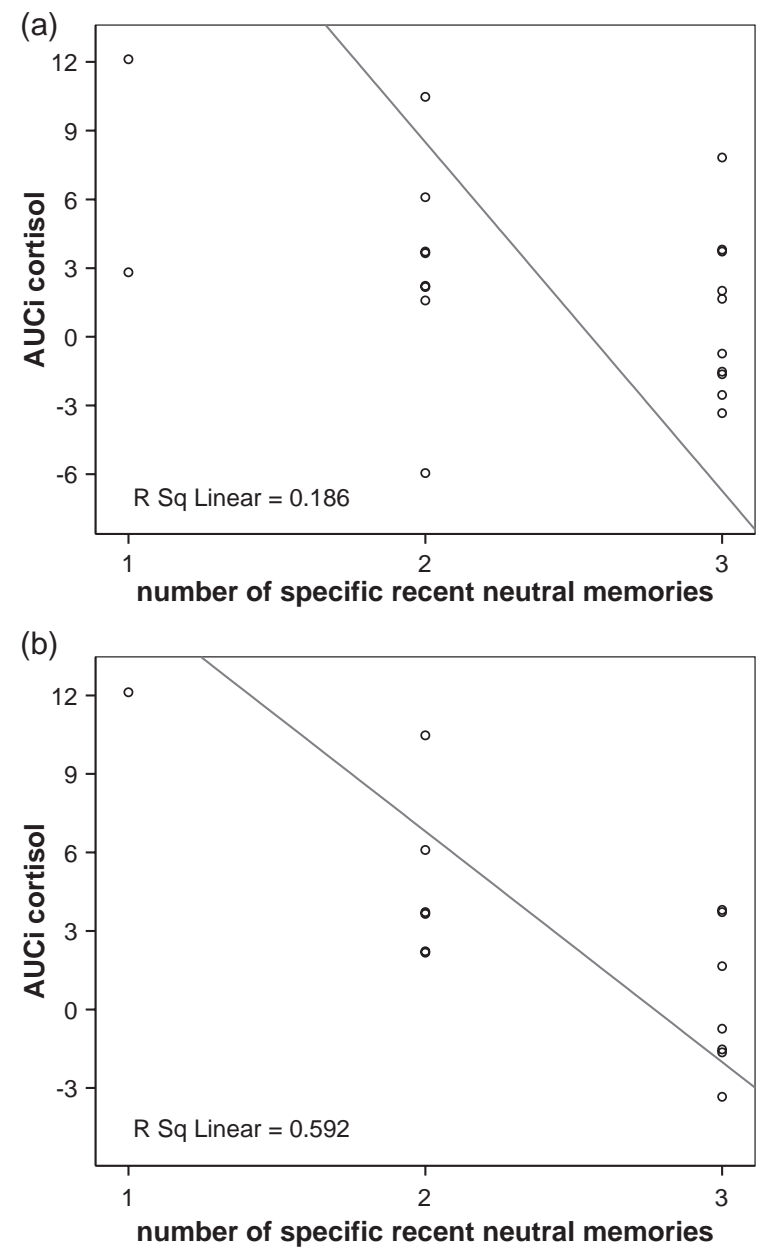

Figure 3. Scatter plots of the correlation between increases of cortisol (AUCi) and specificity of recent memories elicited by neutral cue words in (a) the whole stress group $(N=20)$, and (b) the stress group that still showed heightened SBP at $\mathrm{t}=60(N=14)$.

this sub-group was limited to participants who showed a heightened heart rate and diastolic blood pressure at $\mathrm{t}=60$ as well, only seven participants were left, leaving very low statistical power for correlation analyses, but showing similar results $(r h o=-0.69, p=.08)$. No relations between cortisol increase and any of the other types of memory were found in this these sub-groups.

In the scatter plots of Figure 3 it might seem that the participants that only retrieved one specific recent neutral memory are partly causing the correlations. After removing those participants from the data set, the correlations between cortisol and memory specificity failed to reach significance in the whole stress group but remained significant in the subgroup with heightened systolic blood pressure (whole stress 
group: $r h o=-0.29, \quad p=.26 ;$ subgroup: $r h o=$ $-0.58, p=.04)$.

\section{DISCUSSION}

We found no effects of a psychosocial stressor on autobiographic memory specificity or subjective experience of the memories in healthy young men. This contradicts our expectations based on earlier findings of impaired memory retrieval after stress and cortisol administration, as well as predictions from the CaRFAX model. However, lower specificity of recent, neutral memories tended to be related to a larger increase in cortisol due to stress, especially when participants were physically aroused while retrieving their autobiographical memories. This finding should be interpreted with caution, however, since it was found in a small group and we expected to find correlations of cortisol with memory specificity on negative and remote cues as well.

Several rationales can be put forward to account for our null findings regarding the group differences and the single correlation. First, the increase in endogenous cortisol was much lower than, for example, the pharmacologically induced cortisol increase in the study by Buss et al. (2004). In that study cortisol increased in average from 10.01 to $99.13 \mathrm{nmol} / \mathrm{l}$ after $10 \mathrm{mg}$ of cortisol administration, while in our study cortisol increased on average from 10.25 to $14.28 \mathrm{nmol} / \mathrm{L}$ after the stress task. In addition we used a rough estimate of the cortisol increase during the memory task, as we only measured three time points in total that did not reflect the actual levels during the memory task. The area under the curve reflected not only the increase in cortisol but also the speed of recovery during the memory task as well. A recent review by Het et al. (2005) has also shown that cortisol effects on memory may be strongest in the morning when natural cortisol levels are high due to diurnal rhythms, while the present study was carried out in the afternoon. In addition, endogenous cortisol increases might only affect autobiographical memory retrieval when physical arousal is high (see also Tollenaar et al., 2008). As shown in Figure 1, most sympathetic measures (heart rate and blood pressure) were back to baseline at the end of the autobiographical memory task. In the sub-group of participants who still showed elevated blood pressure levels at the end of the memory task compared to baseline, the association between cortisol increase and the number of specific recent neutral autobiographical memory retrieval was indeed stronger and significant. Furthermore, mood was not affected by the stressor, which might be another reason why we did not find group effects, as could be expected on the basis of the functional avoidance mechanism of the CaRFAX model (see Williams et al., 2007) and earlier findings of mood inductions on autobiographical memory retrieval (Au Yeung et al., 2006; Svaldi \& Mackinger, 2003). However, effects of stress on declarative memory without decreases in mood have been reported before (Domes, Heinrichs, Rimmele, Reichwald, \& Hautzinger, 2004; Tollenaar et al., 2008). Hence it cannot be ruled out that the present stress task did not provide a sufficient stressor or high enough cortisol levels to affect autobiographical memory recall, even though participants were physically aroused by the stress task.

Second, this study was conducted among healthy young men and specificity scores were relatively high. The memory traces might have been too strong to be affected by stress and moderate cortisol increases. Studies performed on clinical populations that are characterised by lowered specificity (over-generality) to start with might show stronger effects of stress and cortisol increases on autobiographical memory. Furthermore, autobiographical memory failures have been linked to the content of the cue words used to elicit autobiographical memories in previously depressed patients (Barnhofer, Crane, Spinhoven, \& Williams, 2007). It is possible that self-referenced cue words trigger more overgeneral answers under stress. This would be in line with the CaRFAX model (Williams et al., 2007) predicting more abstract-conceptual thinking and rumination when self-schemas are activated. In the present study the stress task might not have elicited enough negative self-schemas, or they were no longer activated during the memory task. In addition, the model by Williams and colleagues predicts that increasing cognitive load during memory retrieval might lead to less available executive functioning capacity to retrieve specific memories. Since the memory task was performed after the stress task, executive capacities might have been sufficient again to perform the task.

A third explanation might be that our null findings are due to power problems. We based our group sizes on the large effect of cortisol on autobiographical memory in the study by Buss 
and colleagues (Cohen's $d>1$, group size calculation with GPower 3.0.10), but 20 subjects per group might not have been sufficient to find effects of the stressor, with cortisol increases much lower than in the study by Buss et al. (2004). However, the fact that all $F$-values for the group effects were smaller than 1 indicates that bigger group sizes would probably not have led to significant effects.

While no effect of the stress task was found on autobiographical memories measured with the AMT, we did find interesting differences in subjective experience and specificity of memories, dependent on the remoteness and emotional tone of the cue words used to elicit the autobiographical memories. Both memories elicited by neutral cue words, and memories that were instructed to be remote, were rated as less emotionally intense and were re-experienced less intensively than memories elicited by negative cue words and that were instructed to be from the last 2 years, in line with previous research (e.g., Sutin \& Robins, 2007). Interestingly, neutral memories were recalled with less specificity when these memories were remote in comparison to recent neutral memories. In contrast, the recall of remote negative events was still accompanied by specific details compared to recent negative events, even when the events had taken place a long time ago. The findings that negative memories are re-experienced more intensely than relatively neutral memories, and are still as specific when they are remote as when they are recent, are in line with the common finding that emotionally arousing experiences are generally well remembered. Stress hormones like adrenaline and cortisol, released by emotional arousal, appear to play an important role in enabling the significance of an experience to regulate the strength of the memory of that experience (from McGaugh, 2000).

It is interesting that in previous studies using word tasks cortisol has been found to impair mostly the retrieval of emotional words (for an overview see Wolf, 2008) whereas, so far in studies on autobiographical memories, neutral memories are affected most (see Buss et al., 2004, and data from the present study). Possibly, emotional autobiographical memories are not as sensitive to the effects of cortisol as recently learned emotional words. This is important for clinical practice where cortisol administration is thought to have potentially beneficial effects by blocking the excessive retrieval of emotional (traumatic) memories, leading to fewer intrusive memories and PTSD symptoms (de Quervain \& Margraf, 2008). It should be noted, however, that using the AMT, autobiographical memory retrieval is assessed by the measure of specificity. It is not possible to conclude whether the memories recalled specifically are accurate or are lacking essential information. After the stress task participants may have felt pressure to perform well on the memory task as well, and possibly confabulated autobiographical memories when they could not recall a specific real-life memory. Future studies should therefore try to incorporate accuracy measures of autobiographical memories. Furthermore, in the present study an association was found between cortisol increases and recall of recent neutral autobiographical memories, not remote neutral autobiographical memories. Possibly, recalling recent specific memories is mediated by different brain processes and areas than the recall of remote specific memories, and therefore differently influenced by cortisol. Future studies using functional MRI to study cortisol effects on autobiographical memories could shed more light on this issue.

Finally, stress and mild cortisol elevations might simply not affect autobiographical memory retrieval in healthy young men. The only finding in line with this option comes from a study in depressed patients that also did not find a strong association between higher basal cortisol levels and lessspecific autobiographical memories, but even report inverted relations, with cortisol decreases relating to less-specific autobiographical memories (Barnhofer, Kuehn, \& de Jong-Meyer, 2005). Taken together, this study should be regarded as a very first step in investigating the role of stress exposure and endogenous cortisol increases on autobiographical memories. Given the importance of understanding the impaired autobiographical memory in stress-related disorders, such as depression or PTSD, this is a field that needs to be investigated further. In future studies the effects of stress on memory could be investigated in vulnerable groups, already prone to lowered autobiographical memory specificity. Furthermore, since stress-related disorders are more prevalent in women, female participants should be included in future studies as well, to study the possible differential effects of gender on the relation between stress and autobiographical memory retrieval. Besides specificity, accuracy measures of autobiographical memory should also be included if possible. In addition, our study indicates that differences in remoteness and emotionality of the 
memories are important to take into account when studying the relation among stress, cortisol, and autobiographical memory.

Manuscript received 04 August 2008 Manuscript accepted 24 November 2008 First published online 20 January 2009

\section{REFERENCES}

Au Yeung, C. A., Dalgleish, T., Golden, A. M., \& Schartau, P. (2006). Reduced specificity of autobiographical memories following a negative mood induction. Behaviour Research And Therapy, 44, 1481-1490.

Barnhofer, T., Crane, C., Spinhoven, P., \& Williams, J. M. (2007). Failures to retrieve specific memories in previously depressed individuals: Random errors or content-related? Research And Therapy, 45, 18591869.

Barnhofer, T., Kuehn, E. M., \& de Jong-Meyer, R. (2005). Specificity of autobiographical memories and basal cortisol levels in patients with major depression. Psychoneuroendocrinology, 30, 403-411.

Bryant, R. A., Sutherland, K., \& Guthrie, R. M. (2007). Impaired specific autobiographical memory as a risk factor for posttraumatic stress after trauma. Journal of Abnormal Psychology, 116, 837-841.

Buchanan, T. W., \& Lovallo, W. R. (2001). Enhanced memory for emotional material following stresslevel cortisol treatment in humans. Psychoneuroendocrinology, 26, 307-317.

Buss, C., Wolf, O. T., Witt, J., \& Hellhammer, D. H. (2004). Autobiographic memory impairment following acute cortisol administration. Psychoneuroendocrinology, 29, 1093-1096.

Bywaters, M., Andrade, J., \& Turpin, G. (2004). Determinants of the vividness of visual imagery: The effects of delayed recall, stimulus affect and individual differences. Memory, 12, 479-488.

Conway, M. A., \& Pleydell-Pearce, C. W. (2000). The construction of autobiographical memories in the self-memory system. Psychological Review, 107, 261-288.

Dalgleish, T., Williams, J. M., Golden, A. M., Perkins, N., Barrett, L. F., Barnard, P. J., et al. (2007). Reduced specificity of autobiographical memory and depression: The role of executive control. Journal of Experimental Psychology: General, 136, 23-42.

de Quervain, D. J., Aerni, A., \& Roozendaal, B. (2007). Preventive effect of beta-adrenoceptor blockade on glucocorticoid-induced memory retrieval deficits. American Journal of Psychiatry, 164, 967-969.

de Quervain, D. J. F., Henke, K., Aerni, A., Treyer, V., McGaugh, J. L., Berthold, T., et al. (2003). Glucocorticoid-induced impairment of declarative memory retrieval is associated with reduced blood flow in the medial temporal lobe. European Journal of Neuroscience, 17, 1296-1302.

de Quervain, D. J. F., \& Margraf, J. (2008). Glucocorticoids for the treatment of post-traumatic stress disorder and phobias: A novel therapeutic approach. European Journal of Pharmacology, 583, 365-371.

Domes, G., Heinrichs, M., Rimmele, U., Reichwald, U., \& Hautzinger, M. (2004). Acute stress impairs recognition for positive words: Association with stress-induced cortisol secretion. Stress-The International Journal on the Biology of Stress, 7, 173-181.

Elzinga, B. M., \& Roelofs, K. (2005). Cortisol-induced impairments of working memory require acute sympathetic activation. Behavioral Neuroscience, 119, 98-103.

Greenberg, D. L., Rice, H. J., Cooper, J. J., Cabeza, R., Rubin, D. C., \& LaBar, K. S. (2005). Co-activation of the amygdala, hippocampus and inferior frontal gyrus during autobiographical memory retrieval. Neuropsychologia, 43, 659-674.

Harvey, A. G., Bryant, R. A., \& Dang, S. T. (1998). Autobiographical memory in acute stress disorder. Journal of Consulting and Clinical Psychology, 66, 500-506.

Het, S., Ramlow, G., \& Wolf, O. T. (2005). A metaanalytic review of the effects of acute cortisol administration on human memory. Psychoneuroendocrinology, 30, 771-784.

Kangas, M., Henry, J. L., \& Bryant, R. A. (2005). A prospective study of autobiographical memory and posttraumatic stress disorder following cancer. Journal of Consulting and Clinical Psychology, 73, 293299.

Kirschbaum, C., Pirke, K. M., \& Hellhammer, D. H. (1993). The 'Trier Social Stress Test': A tool for investigating psychobiological stress responses in a laboratory setting. Neuropsychobiology, 28, 76-81.

Kirschbaum, C., Wust, S., \& Hellhammer, D. (1992). Consistent sex-differences in cortisol responses to psychological stress. Psychosomatic Medicine, 54, 648-657.

Kuhlmann, S., Kirschbaum, C., \& Wolf, O. T. (2005a). Effects of oral cortisol treatment in healthy young women on memory retrieval of negative and neutral words. Neurobiology of Learning and Memory, 83, 158-162.

Kuhlmann, S., Piel, M., \& Wolf, O. T. (2005b). Impaired memory retrieval after psychosocial stress in healthy young men. Journal of Neuroscience, 25, 2977-2982.

Lupien, S. J., Gillin, C. J., \& Hauger, R. L. (1999). Working memory is more sensitive than declarative memory to the acute effects of corticosteroids: A dose-response study in humans. Behavioral Neuroscience, 113, 420-430.

Lupien, S. J., \& Lepage, M. (2001). Stress, memory, and the hippocampus: Can't live with it, can't live without it. Behavioural Brain Research, 127, 137158.

McGaugh, J. L. (2000). Neuroscience - memory - a century of consolidation. Science, 287, 248-251.

Oei, N. Y. L., Elzinga, B., Wolf, O. T., de Ruiter, M. B., Damoiseaux, J. S., Kuijer, J. P., et al. (2007). Glucocorticoids decrease hippocampal and prefrontal activation during declarative memory retrieval in young men. Brain Imaging and Behavior, 1, 31-41.

Oei, N. Y. L., Everaerd, W. T. A. M., Elzinga, B. M., van Well, S., \& Bermond, B. (2006). Psychosocial stress 
impairs working memory at high loads: An association with cortisol levels and memory retrieval. Stress-the International Journal on the Biology of Stress, 9, 133-141.

Pruessner, J. C., Kirschbaum, C., Meinlschmid, G., \& Hellhammer, D. H. (2003). Two formulas for computation of the area under the curve represent measures of total hormone concentration versus time-dependent change. Psychoneuroendocrinology, 28, 916-931.

Roozendaal, B., Hahn, E. L., Nathan, S. V., de Quervain, D. J., \& McGaugh, J. L. (2004). Glucocorticoid effects on memory retrieval require concurrent noradrenergic activity in the hippocampus and basolateral amygdala. Journal of Neuroscience, $24,8161-8169$.

Roozendaal, B., McReynolds, J. R., \& McGaugh, J. L. (2004). The basolateral amygdala interacts with the medial prefrontal cortex in regulating glucocorticoid effects on working memory impairment. Journal of Neuroscience, 24, 1385-1392.

Spinhoven, P., Bockting, C. L. H., Kremers, I. P., Schene, A. H., \& Williams, J. M.G. (2007). The endorsement of dysfunctional attitudes is associated with an impaired retrieval of specific autobiographical memories in response to matching cues. Memory, 15, 324-338.

Spinhoven, P., Ormel, J., Sloekers, P. P., Kemper, G. I., Speckens, A. E., \& Van Hemert, A. M. (1997). A validation study of the Hospital Anxiety and Depression Scale (HADS) in different groups of Dutch subjects. Psychologocal Medicine, 27, 363-370.

Sutin, A. R., \& Robins, R. W. (2007). Phenomenology of autobiographical memories: The memory experiences questionnaire. Memory, 15, 390-411.

Svaldi, J. J., \& Mackinger, H. F. (2003). Veranderung der Spezifitat autobiographischer Erinnerungen durch Stimmungsinduktion [Change in the specificity of autobiographical memories through mood induction]. Zeitschrift für Klinische Psychologie, Psychiatrie und Psychotherapie, 51, 144-157.

Tollenaar, M. S., Elzinga, B. M., Spinhoven, P., \& Everaerd, W. A. (2008). The effects of cortisol increase on long-term memory retrieval during and after acute psychosocial stress. Acta Psychologica (Amst), 127, 542-552.

Tulving, E. (2002). Episodic memory: From mind to brain. Annual Review of Psychology, 53, 1-25.

van Aken, M. O., Romijn, J. A., Miltenburg, J. A., \& Lentjes, E. G. W. M. (2003). Automated measurement of salivary cortisol. Clinical Chemistry, 49, 1408-1409.

van der Does, A. J. W. (2002). BDI-II-NL Handleiding. De Nederlandse versie van de Beck Depression Inventory-second edition [BDI-II Dutch manual]. Lisse, The Netherlands: Swets \& Zeitlinger.

Williams, J. M., \& Scott, J. (1988). Autobiographical memory in depression. Psychological Medicine, 18, 689-695.

Williams, J. M. G., Barnhofer, T., Crane, C., Hermans, D., Raes, F., Watkins, E., et al. (2007). Autobiographical memory specificity and emotional disorder. Psychological Bulletin, 133, 122-148.

Williams, J. M. G., \& Broadbent, K. (1986). Autobiographical memory in suicide attempters. Journal of Abnormal Psychology, 95, 144-149.

Wolf, O. T. (2008). The influence of stress hormones on emotional memory: Relevance for psychopathology. Acta Psychologica (Amst), 127, 513-531.

Wolf, O. T., Convit, A., McHugh, P. F., Kandil, E., Thorn, E. L., De Santi, S., et al. (2001). Cortisol differentially affects memory in young and elderly men. Behavioral Neuroscience, 115, 1002-1011.

\section{Appendix}

Words used in the AMT (translated from Dutch)

\begin{tabular}{ll}
\hline Negative & Neutral \\
\hline grief & grass \\
regret & bread \\
ashamed & bathe \\
bad & nature \\
hurt & library \\
guilt & fast \\
\hline
\end{tabular}

